\title{
DUALITY FOR A COHEN-MACAULAY LOCAL RING
}

\author{
MOHAMMAD ALI ESMKHANI AND MASSOUD TOUSI
}

\begin{abstract}
Let $(R, \mathfrak{m})$ be a Cohen-Macaulay local ring. If $R$ has a canonical module, then there are some interesting results about duality for this situation. In this paper, we show that one can indeed obtain similar these results in the case $R$ has not a canonical module. Also, we give some characterizations of complete big Cohen-Macaulay modules of finite injective dimension and by using it some characterizations of Gorenstein modules over the $\mathfrak{m}$-adic completion of $R$ are obtained.
\end{abstract}

\section{INTRODUCTION}

Throughout the paper, $R$ will denote a commutative Noetherian local ring with nonzero identity and maximal ideal $\mathfrak{m}$. Unless stated otherwise, the notation will be the same as used in [2]. When discussing the completion of $R$ or a module over $R$, we will mean the $\mathfrak{m}$-adic completion.

The canonical module of a Cohen-Macaulay ring gives a useful technical tool in commutative algebra, algebraic geometry, and singularity theory via various duality theorems. Grothendieck defined the notion of a module of dualizing differentials for a complete local ring and proved some results on local duality for complete local rings and some properties of a module of dualizing differentials, see [7]. In [8], the theory of canonical modules for a general Noetherian local ring $R$ was studied. We recall the definition of the canonical modules of a Noetherian local ring $(R, \mathfrak{m})$ of dimension $n$. A finitely generated $R$-module $C$ is called the canonical module of $R$ if $C \otimes_{R} \hat{R} \cong \operatorname{Hom}_{R}\left(H_{\mathfrak{m}}^{n}(R), E_{R}(R / \mathfrak{m})\right)$, see [8]. There are many unknowns about canonical modules over general local rings, existence theorems are among those. In [10], Reiten (and independently Foxby [5]) proved that a Cohen-Macaulay local ring has a canonical module if and only if it is a homomorphic image of a Gorenstein

2000 Mathematics Subject Classification. Primary 13C14, 13J10; Secondary 13D05, 13C13.

Key words and phrases. Canonical module, balanced big Cohen-Macaulay module, CohenMacaulay local ring, complete module, Cousin complex.

This research was in part supported by a grant from IPM (No. 87130214). 
local ring. Therefore, there exists a Cohen-Macaulay local ring which does not have canonical modules. It is known that any two canonical modules of $R$ are isomorphic. Also, it is known that over a Cohen-Macaulay local ring $R$, a maximal Cohen-Macaulay module $C$ of type 1 and of finite injective dimension is a canonical module of $R$.

Section 2 develops a variation of Grothendieck duality, namely duality over a Cohen-Macaulay local ring. Let $R$ be a Cohen-Macaulay local ring and $K$ the canonical module of the completion of $R$. The $R$-module $K$ is not finitely generated in general. Many facts that are valid for finitely generated modules are not valid for $K$. But our achievement in this paper is that, $K$ as an $R$-module has many properties similar to canonical modules. We do this by applying some results of H.B. Foxby [6], A.M. Simon [18] and R.Y. Sharp [15]. Simon [18] has established analogies between complete modules and finitely generated ones. The purpose of [15] is to show that balanced big Cohen-Macaulay modules (over an arbitrary local ring $R$ ) have many of the properties of maximal Cohen-Macaulay modules. Foxby [6] has established some properties of the depth of finitely generated modules for a non-finitely generated modules.

One of the main result of this paper is

Theorem A (See Theorem 2.9) (Duality) Let $(R, \mathfrak{m})$ be a Cohen-Macaulay local ring of dimension $d, K$ the canonical module of the completion of $R$, and $\mathrm{D}$ the functor $\operatorname{Hom}_{R}(-, K)$. Then

i) D takes balanced big Cohen-Macaulay R-module to balanced big Cohen-Macaulay $R$-module.

ii) D takes exact sequence of balanced big Cohen-Macaulay $R$-modules to exact sequence.

iii) There exists a natural transformation $\tau:-\otimes_{R} \hat{R} \longrightarrow \operatorname{Hom}_{R}\left(\operatorname{Hom}_{R}(-, K), K\right)$ such that $\tau_{M}$ sending $m \otimes \hat{r} \in M \otimes_{R} \hat{R}$ to the map $\alpha \longmapsto \hat{r} \alpha(m)$ for $\alpha \in \operatorname{Hom}_{R}(M, K)$ is an isomorphism when $M$ is a maximal Cohen-Macaulay $R$-module.

The purpose of Section 3 and 4 is to present some characterizations of $K$. We do this by characterizing the Gorenstein modules over $\hat{R}$. Gorenstein $R$-modules are those finitely generated $R$-modules for which the Cousin complex for $M$ with respect 
to $M$-height filtration provides a minimal injective resolution (see [13]). R.Y. Sharp obtained various characterizations and properties of Gorenstein modules in $[\mathbf{1 3}$, Theorem 3.1]. Note that, it is shown that in [14] that any Gorenstein $\hat{R}$-module is a finite sum of copies of $K$. First, we obtain some characterizations for complete big Cohen-Macaulay modules (see Proposition 3.2). For complete big Cohen-Macaulay modules of finite injective dimension a better characterization is provided:

Theorem B (See Theorem 3.4) Let $(R, \mathfrak{m})$ be a Noetherian local ring and $M a$ nonzero complete $R$-module. Then the following conditions are equivalent:

i) $\mathrm{ht}_{M}(\mathfrak{m})=\operatorname{dim}(R)$ and for every $\mathfrak{p} \in \operatorname{supp}_{R}(M), \mu_{R}^{i}(\mathfrak{p}, M) \neq 0$ if and only if $i \neq \mathrm{ht}_{M_{\mathfrak{p}}}\left(\mathfrak{p} R_{\mathfrak{p}}\right)$.

ii) $\mu_{R}^{i}(\mathfrak{m}, M)=0$ if and only if $i \neq \operatorname{dim}(R)$.

iii) $M$ is a big Cohen-Macaulay $R$-module with respect to a system of parameters for $R$ and $M$ has finite injective dimension.

iv) $\operatorname{depth}_{R}(M)=\operatorname{dim}(R)$ and $M$ has finite injective dimension.

v) $\operatorname{injdim}_{R}(M)=\operatorname{depth}_{R}(M)=\operatorname{dim}_{R}(M)=\operatorname{dim}(R)=\operatorname{depth}(R)$.

Finally, we establish the following theorem

Theorem C (See Theorem 4.2) Let $(R, \mathfrak{m})$ be a Noetherian local ring of dimension $d$ and $M$ a nonzero $R$-module. Then the following conditions are equivalent:

i) $M$ is complete and big Cohen-Macaulay with respect to a system of parameters $x_{1}, \ldots, x_{d}$ such that $\operatorname{injdim}_{R} M$ and $\mu_{R}^{d}(\mathfrak{m}, M)$ are finite.

ii) $M$ is a Gorenstein $\hat{R}$-module.

iii) $M$ is complete and for all Cohen-Macaulay $R$-module $L$ of dimension $t$, we have

a) $\operatorname{Ext}_{R}^{i}(L, M)=0$ for all $i \neq d-t$, and

b) $\operatorname{Ext}_{R}^{d-t}\left(\operatorname{Ext}_{R}^{d-t}(L, M), M\right) \cong L \otimes_{R} F$, where $F$ is a finitely generated free $\hat{R}$-module.

\section{The Canonical modules of the completion of a Cohen-Macaulay} LOCAL RING

We begin this section, by recalling the definitions of the notion of depth, dimension of an $R$-module $M$, and balanced big Cohen-Macaulay modules (recall that $M$ is not assumed to be finitely generated). 
Definition and Remark 2.1. Let $(R, \mathfrak{m})$ be a Noetherian local ring.

i) If $M$ is an $R$-module, we define the dimension of $M$ by

$$
\operatorname{dim}_{R}(M)=\operatorname{dim}_{R}\left(R / \operatorname{Ann}_{R}(M)\right) .
$$

By the height $\operatorname{ht}_{M}(\mathfrak{m})$ of an $R$-module $M$ we mean the dimension of the support $\operatorname{Supp}_{R}(M)$ of $M$.

ii) In $[\mathbf{6}$, Section 1$]$, Foxby has established the concept of depth to any $R$-module $M$ by means of the formula

$$
\operatorname{depth}_{R}(M)=\inf \left\{i \geq 0 \mid \operatorname{Ext}_{R}^{i}(R / \mathfrak{m}, M) \neq 0\right\} .
$$

For a nonzero $M$ which is not finitely generated, it is possible for this depth to be $\infty$. If the $R$-module $M$ satisfies $\mathfrak{m} M \neq M$, then $\operatorname{depth}_{R}(M) \leq \mathrm{ht}_{M}(\mathfrak{m})<\infty$ (see [6, Corollary 2.2]).

iii) (See [19, Definition 5.3.1]) Let $M$ be an $R$-module with $\mathfrak{m} M \neq M$. Then $\mathrm{dp}_{R}(M)$ is defined to be the supremum of the lengths of all $M$-sequence contained in $\mathfrak{m}$. Since $R$ is Noetherian, $\operatorname{dp}_{R}(M)<\infty$. It is well known that if $M$ is nonzero finitely generated, then $\operatorname{dp}_{R}(M)=\operatorname{depth}_{R}(M)$.

iv) Let $M$ be an $R$-module with $\mathfrak{m} M \neq M$. Then, by ii) and [19, Proposition 5.3 .7 (ii)],

$$
\operatorname{dp}_{R}(M) \leq \operatorname{depth}_{R}(M) \leq \operatorname{ht}_{M}(\mathfrak{m}) \leq \operatorname{dim}_{R}(M) \leq \operatorname{dim}(R)
$$

v) Let $a_{1}, \ldots, a_{n}$ be a system of parameters (s.o.p.) for $R$. A (not necessarily finitely generated) $R$-module $M$ is said to be a big Cohen-Macaulay $R$-module with respect to $a_{1}, \ldots, a_{n}$ if $a_{1}, \ldots, a_{n}$ is an $M$-sequence, that is if $M \neq\left(a_{1}, \ldots, a_{n}\right) M$ and, for each $i=1, \ldots, n,\left(\left(a_{1}, \ldots, a_{i-1}\right) M: a_{i}\right)=\left(a_{1}, \ldots, a_{i-1}\right) M$. It will be convenient to use abbreviation b.C.M. for big Cohen-Macaulay.

vi) Let $a_{1}, \ldots, a_{n}$ be a system of parameters for $R$ and let $M$ be b.C.M. $R$-module with respect to $a_{1}, \ldots, a_{n}$. Then there exists $t \in \mathrm{N}$ such that $\mathfrak{m}^{t} \subseteq\left(a_{1}, \ldots, a_{n}\right) R$ and $a_{1}, \ldots, a_{n}$ is an $M$-sequence. So, $\mathfrak{m} M \neq M$. Hence, by iv), we have

$$
\operatorname{dp}_{R}(M)=\operatorname{depth}_{R}(M)=\operatorname{ht}_{M}(\mathfrak{m})=\operatorname{dim}_{R}(M)=\operatorname{dim}(R) .
$$

vii) An $R$-module $M$ is called balanced big Cohen-Macaulay if every system of parameter for $R$ is an $M$-sequence (see [15, Definition 1.4]). It will be convenient to use abbreviation b.b.C.M. for balanced big Cohen-Macaulay. In $[\mathbf{1 5 , 1 6}]$, it is 
shown that b.b.C.M. modules verify some of the properties of finitely generated Cohen-Macaulay modules.

Lemma 2.2. Let $(R, \mathfrak{m})$ be a Cohen-Macaulay local ring and $K$ the canonical module of $\hat{R}$. Then

i) $K$ is complete with respect to $\mathfrak{m}$-adic topology.

ii) $K$ is a b.b.C.M. R-module.

iii) $\operatorname{inj}_{\operatorname{dim}_{R}} K<\infty$.

Proof. i) The natural $R$-homomorphism $\theta: K \longrightarrow \hat{K}$ is an isomorphism. This follows from the fact that $\mathfrak{m}^{n} K=(\mathfrak{m} \hat{R})^{n} K$ for all $n \geq 0$.

ii) Let $x_{1}, \ldots, x_{d}$ be a system of parameters for $R$ and let $\psi: R \longrightarrow \hat{R}$ be the natural ring homomorphism. Then $\psi\left(x_{1}\right), \ldots, \psi\left(x_{d}\right)$ is an $\hat{R}$-sequence, because $R$ is Cohen-Macaulay. On the other hand, $K$ is a maximal Cohen-Macaulay $\hat{R}$-module, and so $x_{1}, \ldots, x_{d}$ is a $K$-sequence.

iii) This follows from the fact that any injective $\hat{R}$-module is injective as an $R$ module. Note that the injective dimension ok $K$ as an $\hat{R}$-module is finite.

Simon [18] has shown that complete modules behave similar to finitely generated modules in many respects. We summarize some important properties of complete modules from $[\mathbf{1 8}]$ in the following remark.

Remark 2.3. Let $(R, \mathfrak{m})$ be a Noetherian local ring, and let $M$ and $N$ be two complete $R$-modules. Then

i) If $f: N \longrightarrow M$ is a morphism between $R$-modules such that $M=f(N)+\mathfrak{m} M$, then $f$ is surjective.

ii) For each $R$-module $X$, if $\operatorname{Ext}_{R}^{i}(X, M) \neq 0$, then $\operatorname{Ext}_{R}^{i}(X, M) \neq \operatorname{mExt}_{R}^{i}(X, M)$.

iii) $\operatorname{injdim}_{R}(M)=\sup \left\{i \mid \operatorname{Ext}_{R}^{i}(R / \mathfrak{m}, M) \neq 0\right\}$.

iv) If $M$ has finite injective dimension, then $\operatorname{injdim}_{R}(M)=\operatorname{depth} R$.

v) If $R$ has a big Cohen-Macaulay module $C$ and a complete module $M$ of finite injective dimension, then the $\operatorname{ring} R$ is Cohen-Macaulay.

To prove Theorem 2.9, we need the following five results.

Lemma 2.4. Let $(R, \mathfrak{m})$ be a Noetherian local ring and $M$ an $R$-module of dimension $t \geq 1$ with $\mathfrak{m} M \neq M$ ( $M$ not necessarily finitely generated). If there exists an $M$ sequence $x_{1}, \ldots, x_{t}$, then

$$
\operatorname{dim}_{R} M=\operatorname{dim}_{R}\left(M / x_{1} M\right)+1 .
$$


Proof. It is clear that $x_{1} \in R \backslash \mathrm{Z}_{R}(M)$ implies that $x_{1} \in R \backslash \mathrm{Z}_{R}\left(R / \operatorname{Ann}_{R} M\right)$. Hence, we have

$$
\begin{aligned}
\operatorname{depth}_{R}\left(M / x_{1} M\right) \leq \operatorname{dim}_{R}\left(R /\left(\operatorname{Ann}_{R}\left(M / x_{1} M\right)\right)\right) & \leq \operatorname{dim}_{R}\left(R /\left(\operatorname{Ann}_{R} M+x_{1} R\right)\right) \\
& =\operatorname{dim}_{R}\left(\left(R / \operatorname{Ann}_{R} M\right) / x_{1}\left(R / \operatorname{Ann}_{R} M\right)\right) \\
& =\operatorname{dim}_{R}\left(R / \operatorname{Ann}_{R} M\right)-1 \\
& =t-1,
\end{aligned}
$$

where the first inequality follows from $[\mathbf{6}$, Corollary 2.2]. On the other hand, by $[\mathbf{2}$, Proposition 1.2.3 and Lemma 1.2.4], $\operatorname{Ext}_{R}^{i}\left(R / \mathfrak{m}, M / x_{1} M\right)=0$ for all $0 \leq i \leq t-2$. So $\operatorname{depth}_{R}\left(M / x_{1} M\right) \geq t-1$. Thus, the assertion follows.

Proposition 2.5. Let $(R, \mathfrak{m})$ be a Noetherian local ring.

i) Let $C$ be a complete $R$-module of injective dimension s. If $M$ is an $R$-module and $x_{1}, x_{2}, \ldots, x_{l}$ an $M$-sequence, then $\operatorname{Ext}_{R}^{j}(M, C)=0$ for $j>s-l$.

ii) Let $(R, \mathfrak{m})$ be a Cohen-Macaulay local ring of dimension d and $C$ a b.b.C.M. $R$-module. If $M$ is an $R$-module of dimension $t$, then $\operatorname{Ext}_{R}^{j}(M, C)=0$ for all $j<d-t$.

iii) Let $(R, \mathfrak{m})$ be a Cohen-Macaulay local ring of dimension d, $C$ a complete $R$ module of finite injective dimension which is b.b.C.M. and $M$ an R-module of dimension $t \geq 0$. Let $x_{1}, x_{2}, \ldots, x_{t}$ be an $M$-sequence. If $\mathfrak{m} M \neq M$, then $\operatorname{Ext}_{R}^{d-t}(M, C) \neq 0$, $x_{1}, x_{2}, \ldots, x_{t}$ is an $\operatorname{Ext}_{R}^{d-t}(M, C)$-sequence and the dimension of $\operatorname{Ext}_{R}^{d-t}(M, C)$ is equal to $t$.

Proof. i) The proof is same as $[\mathbf{2}, 3.3 .3(\mathrm{~b})]$, by replacing Nakayama's lemma by Remark 2.3 ii).

ii) We set $\mathfrak{a}=\operatorname{Ann}_{R} M$. Since $R$ is Cohen-Macaulay, we have

$$
\operatorname{ht}_{R}(\mathfrak{a})=\operatorname{dim} R-\operatorname{dim} R / \mathfrak{a}=d-t .
$$

Hence $\operatorname{grade}_{R}(\mathfrak{a})=d-t$, and so there exist $x_{1}, x_{2}, \ldots, x_{d-t}$ in $\mathfrak{a}$ which is an $R$ sequence. Consequently, $x_{1}, x_{2}, \ldots, x_{d-t}$ is a $C$-sequence in $\mathfrak{a}$. Therefore, by $[\mathbf{2}$, Proposition 1.2.3 and Lemma 1.2.4], we have $\operatorname{Ext}_{R}^{j}(M, C)=0$ for all $j<d-t$.

iii) We set $\operatorname{Ext}_{R}^{d-t}(M, C)=N$ and use induction on $t$. Let $t=0$. There exists a system of parameters $y_{1}, y_{2}, \ldots, y_{d}$ of $R$ in $\operatorname{Ann}_{R}(M)$. So, by [2, Lemma 1.2.4],

$$
\operatorname{Ext}_{R}^{d}(M, C) \cong \operatorname{Hom}_{R}\left(M, C /\left(y_{1}, y_{2}, \ldots, y_{d}\right) C\right) .
$$


Since $C /\left(y_{1}, y_{2}, \ldots, y_{d}\right) C \neq 0$ and $\left(y_{1}, y_{2}, \ldots, y_{d}\right) R \subseteq \operatorname{Ann}_{R}\left(C /\left(y_{1}, y_{2}, \ldots, y_{d}\right) C\right)$, we have $\mathfrak{m} \in \operatorname{Ass}_{R}\left(C /\left(y_{1}, y_{2}, \ldots, y_{d}\right) C\right)$. Hence, there is an $R$-monomorphism $\varphi: R / \mathfrak{m} \longrightarrow C /\left(y_{1}, y_{2}, \ldots, y_{d}\right) C$. By viewing $M / \mathfrak{m} M$ as a nonzero vector space over $R / \mathfrak{m}$, we deduce that there is an $R$-epimorphism $\psi: M / \mathfrak{m} M \longrightarrow R / \mathfrak{m}$. Hence $\varphi \psi \pi: M \longrightarrow C /\left(y_{1}, y_{2}, \ldots, y_{d}\right) C$ is a nonzero homomorphism in which $\pi: M \longrightarrow M / \mathfrak{m} M$ is the natural $R$-epimorphism. So, $\operatorname{Ext}_{R}^{d}(M, C) \neq 0$. Also, $\operatorname{Ann}_{R} M \subseteq \operatorname{Ann}_{R} N$. Therefore, $\operatorname{dim}_{R} N=0$.

Now suppose, inductively that $t>0$. It is clear that $\mathfrak{m}\left(M / x_{1} M\right) \neq\left(M / x_{1} M\right)$. Now by Lemma 2.4 $\operatorname{dim}_{R}\left(M / x_{1} M\right)=t-1$. In view of i), ii) and the fact that $\operatorname{injdim}_{R}(C)=\operatorname{depth}(R)=\operatorname{dim}(R)$, the exact sequence $0 \longrightarrow M \stackrel{x_{1}}{\longrightarrow} M \longrightarrow$ $M / x_{1} M \longrightarrow 0$ induces the exact sequence

$$
0 \longrightarrow N \stackrel{x_{1}}{\longrightarrow} N \longrightarrow \operatorname{Ext}_{R}^{d-t+1}\left(M / x_{1} M, C\right) \longrightarrow 0 .
$$

So that, by inductive hypothesis, $\operatorname{Ext}_{R}^{d-t+1}\left(M / x_{1} M, C\right) \neq 0, x_{1}, x_{2}, \ldots, x_{t}$ is an $N$ sequence and the dimension of $\operatorname{Ext}_{R}^{d-t+1}\left(M / x_{1} M, C\right)$ is $t-1$. Also, since $N \neq 0$, it follows from Remark 2.3 ii) that $\mathfrak{m} N \neq N$. So, by Lemma $2.4, \operatorname{dim}_{R} N=t$, because $\operatorname{dim}_{R}\left(N / x_{1} N\right)=t-1$.

In the following we state a result without proof, since the proof is same as that of [3, Proposition 21.12].

Lemma 2.6. Let $(R, \mathfrak{m})$ be a Noetherian local ring of dimensiond, and $C$ a b.b.C.M. $R$-module. If $M$ is an $R$-module and $\operatorname{Ext}_{R}^{1}(M, C)=0$, then for any $R$-sequence $x$ we have

$$
\operatorname{Hom}_{R}(M, C) / x \operatorname{Hom}_{R}(M, C) \cong \operatorname{Hom}_{R / x R}(M / x M, C / x C)
$$

by the homomorphism taking the class of map $\varphi: M \longrightarrow C$ to the map $M / x M \longrightarrow$ $C / x C$ induced by $\varphi$.

Lemma 2.7. Let $(R, \mathfrak{m})$ be a Noetherian local ring, and let $M$ and $N$ be two complete $R$-modules. Suppose that $x \in \mathfrak{m}$ is a nonzero-divisor on $M, \varphi: N \rightarrow M$ an $R$ homomorphism and $\bar{\varphi}: N / x N \rightarrow M / x M$ is the R-homomorphism induced by $\varphi$. Then

i) If $\bar{\varphi}$ is an epimorphism, then $\varphi$ is an epimorphism.

ii) If $\bar{\varphi}$ is a monomorphism, then $\varphi$ is a monomorphism. 
Proof. i) If $\bar{\varphi}$ is an epimorphism, then $\varphi(N)+x M=M$. The assertion follows from Remark 2.3 i).

ii) Suppose that $\bar{\varphi}$ is a monomorphism, and let $K=\operatorname{ker} \varphi$. Then $x K=K$, by same argument to the proof of [3, Proposition 21.13]. So $K=\bigcap_{n=0}^{\infty} x^{n} K \subseteq \bigcap_{n=0}^{\infty} x^{n} N$. But, since $N$ is a complete $R$-module, we have $\bigcap_{n=0}^{\infty} x^{n} N=0$, and so $K=0$.

Proposition 2.8. Let $(R, \mathfrak{m})$ be a Cohen-Macaulay local ring of dimension $d$ and $K$ the canonical module of the completion of $R$. The following assertions hold.

i) $\operatorname{Hom}_{R}\left(H_{\mathfrak{m}}^{d}(R), E_{R}(R / \mathfrak{m})\right) \cong K$.

ii) $H_{\mathfrak{m}}^{d}(K) \cong E_{R}(R / \mathfrak{m})$.

iii) There is an $R$-isomorphism $\psi: \hat{R} \longrightarrow \operatorname{Hom}_{R}(K, K)$ which is such that $\psi(\hat{r})=$ $\hat{r} \mathrm{id}_{K}$, for any $\hat{r} \in \hat{R}$.

Proof. i) By using the Flat Base Change Theorem for local cohomology, we obtain

$$
\begin{aligned}
K & \cong \operatorname{Hom}_{\hat{R}}\left(H_{\mathfrak{m} \hat{R}}^{d}(\hat{R}), E_{\hat{R}}(\hat{R} / \mathfrak{m} \hat{R})\right) \\
& \cong \operatorname{Hom}_{\hat{R}}\left(H_{\mathfrak{m}}^{d}(R) \otimes_{R} \hat{R}, E_{\hat{R}}(\hat{R} / \mathfrak{m} \hat{R})\right) \\
& \cong \operatorname{Hom}_{R}\left(H_{\mathfrak{m}}^{d}(R), \operatorname{Hom}_{\hat{R}}\left(\hat{R}, E_{\hat{R}}(\hat{R} / \mathfrak{m} \hat{R})\right)\right. \\
& \cong \operatorname{Hom}_{R}\left(H_{\mathfrak{m}}^{d}(R), E_{R}(R / \mathfrak{m})\right) .
\end{aligned}
$$

ii) In view of the Independence Theorem for local cohomology and $[\mathbf{1}$, Theorem 11.2.8], we have $H_{\mathfrak{m}}^{d}(K) \cong H_{\mathfrak{m} \hat{R}}^{d}(K) \cong E_{\hat{R}}(\hat{R} / \mathfrak{m} \hat{R}) \cong E_{R}(R / \mathfrak{m})$.

iii) We have

$$
\begin{aligned}
\operatorname{Hom}_{R}(K, K) & \cong \operatorname{Hom}_{R}\left(K, \operatorname{Hom}_{R}\left(H_{\mathfrak{m}}^{d}(R), E_{R}(R / \mathfrak{m})\right)\right) \\
& \cong \operatorname{Hom}_{R}\left(K \otimes_{R} H_{\mathfrak{m}}^{d}(R), E_{R}(R / \mathfrak{m})\right) \\
& \cong \operatorname{Hom}_{R}\left(H_{\mathfrak{m}}^{d}(K), E_{R}(R / \mathfrak{m})\right) \\
& \cong \operatorname{Hom}_{R}\left(E_{R}(R / \mathfrak{m}), E_{R}(R / \mathfrak{m})\right) \\
& \cong \hat{R}
\end{aligned}
$$

Here the third isomorphism comes from [1, Ex.6.1.9]. So, $\operatorname{Hom}_{R}(K, K)$ is complete.

Now, we prove this part by induction on $d=\operatorname{dim} R$. When $d=0, R$ is complete, and so we have the result by $[\mathbf{2}$, Theorem 3.3.4(d)]. Now suppose that $d>0$ and the result has been proved for the smaller values. Let $x \in \mathfrak{m}$ be a nonzero-divisor on $R$. Then the module $K / x K$ is the canonical module of $\hat{R} / x \hat{R}$. Also, there exists 
a natural isomorphism $\hat{R} / x \hat{R} \cong(\widehat{R / x R})$. Hence, by the inductive hypothesis, we have an $R / x R$-isomorphism

$$
\theta: \hat{R} / x \hat{R} \longrightarrow \operatorname{Hom}_{R / x R}(K / x K, K / x K)
$$

which is such that $\theta(\hat{r}+x \hat{R})=\hat{r} \operatorname{id}_{K / x K}$, for all $\hat{r} \in \hat{R}$. It follows from Lemma 2.2, Remark 2.3 and Proposition 2.5 i) that $\operatorname{Ext}_{R}^{1}(K, K)=0$. So, by Lemma 2.2 ii) and Lemma 2.6, we have an $R$-isomorphism

$$
f: \operatorname{Hom}_{R}(K, K) / x \operatorname{Hom}_{R}(K, K) \longrightarrow \operatorname{Hom}_{R / x R}(K / x K, K / x K)
$$

by the homomorphism taking the class $\varphi: K \longrightarrow K$ to the map $K / x K \longrightarrow K / x K$ induced by $\varphi$. Let $\bar{\psi}: \hat{R} / x \hat{R} \longrightarrow \operatorname{Hom}_{R}(K, K) / x \operatorname{Hom}_{R}(K, K)$ be the homomorphism induced by $\psi$. It is easy to see that $\theta=f \bar{\psi}$. Hence $\bar{\psi}$ is an $R$-isomorphism. The assertion follows from Lemma 2.7.

Now, we are ready to prove one of the main results of this paper.

Theorem 2.9. (Duality) Let $(R, \mathfrak{m})$ be a Cohen-Macaulay local ring of dimension $d, K$ the canonical module of the completion of $R$, and $\mathrm{D}$ the functor $\operatorname{Hom}_{R}(-, K)$. Then

i) D takes b.b.C.M. R-module to b.b.C.M. R-module.

ii) $D$ takes exact sequence of b.b.C.M. R-modules to exact sequence.

iii) There exists a natural transformation $\tau:-\otimes_{R} \hat{R} \longrightarrow \operatorname{Hom}_{R}\left(\operatorname{Hom}_{R}(-, K), K\right)$ such that $\tau_{M}$ sending $m \otimes \hat{r} \in M \otimes_{R} \hat{R}$ to the map $\alpha \longmapsto \hat{r} \alpha(m)$ for $\alpha \in \operatorname{Hom}_{R}(M, K)$ is an isomorphism when $M$ is a maximal Cohen-Macaulay $R$-module.

Proof. i) Let $M$ be a b.b.C.M. $R$-module. Then, by Remark and definition $2.1 \mathrm{vi}), \mathfrak{m} M \neq M$ and $\operatorname{dim}_{R}(M)=d$. So, the claim follows from Lemma 2.2 and Proposition 2.5 iii).

ii) If $0 \longrightarrow M^{\prime} \longrightarrow M \longrightarrow M^{\prime \prime} \longrightarrow 0$ is an exact sequence of b.b.C.M. $R$-modules, then we get an exact sequence

$$
0 \longrightarrow \operatorname{Hom}_{R}\left(M^{\prime \prime}, K\right) \longrightarrow \operatorname{Hom}_{R}(M, K) \longrightarrow \operatorname{Hom}_{R}\left(M^{\prime}, K\right) \longrightarrow \operatorname{Ext}_{R}^{1}\left(M^{\prime \prime}, K\right) .
$$

Using Lemma 2.2, Remark $2.3 \mathrm{iv})$ and Proposition $2.5 \mathrm{i})$, we obtain $\operatorname{Ext}_{R}^{1}\left(M^{\prime \prime}, K\right)=$ 0 . So, we have the exact sequence

$$
0 \longrightarrow D\left(M^{\prime \prime}\right) \longrightarrow D(M) \longrightarrow D\left(M^{\prime}\right) \longrightarrow 0 .
$$


iii) By Proposition 2.8 iii), there is an isomorphism $\psi: \hat{R} \longrightarrow \operatorname{Hom}_{R}(K, K)$ which is such that $\psi(\hat{r})=\hat{r} \mathrm{id}_{K}$ for any $\hat{r} \in \hat{R}$. Also, we know that there is the natural transformation of functors $\sigma:-\otimes_{R} \operatorname{Hom}_{R}(K, K) \longrightarrow \operatorname{Hom}_{R}\left(\operatorname{Hom}_{R}(-, K), K\right)$ such that for each $R$-module $M,\left(\sigma_{M}(m \otimes f)\right)(g)=f(g(m))$, for $m \in M, f \in$ $\operatorname{Hom}_{R}(K, K)$, and $g \in \operatorname{Hom}_{R}(M, K)$ (see [1, Lemma 10.2.16]). We define a natural transformation of functors

$$
\tau:-\otimes_{R} \hat{R} \longrightarrow \operatorname{Hom}_{R}\left(\operatorname{Hom}_{R}(-, K), K\right)
$$

which is such that for each $R$-module $M, \tau_{M}=\sigma_{M}\left(\mathrm{id}_{M} \otimes_{R} \psi\right)$. Let $M$ be a maximal Cohen-Macaulay $R$-module and let $F_{1} \stackrel{g}{\longrightarrow} F_{0} \stackrel{f}{\longrightarrow} M \longrightarrow 0$ be an exact sequence where $F_{0}$ and $F_{1}$ are finitely generated free $R$-modules.

We consider the following cases:

Case $1 . \operatorname{ker}(f) \neq 0, \operatorname{ker}(g) \neq 0$. We consider two exact sequences

$$
\begin{gathered}
0 \longrightarrow \operatorname{ker}(f) \stackrel{\rho}{\longrightarrow} F_{0} \stackrel{f}{\longrightarrow} M \longrightarrow 0, \\
0 \longrightarrow \operatorname{ker}(g) \stackrel{\mu}{\longrightarrow} F_{1} \stackrel{g}{\longrightarrow} \operatorname{ker}(f) \longrightarrow 0 .
\end{gathered}
$$

It is easy to see that $\operatorname{ker}(f)$ and $\operatorname{ker}(g)$ are maximal Cohen-Macaulay $R$-modules. By the above facts, i) and ii), we obtain the following two exact sequences

$$
0 \longrightarrow \mathrm{D}(\mathrm{D}(\operatorname{ker}(f))) \longrightarrow \mathrm{D}\left(\mathrm{D}\left(F_{0}\right)\right) \longrightarrow \mathrm{D}(\mathrm{D}(M)) \longrightarrow 0
$$

and

$$
0 \longrightarrow \mathrm{D}(\mathrm{D}(\operatorname{ker}(g))) \longrightarrow \mathrm{D}\left(\mathrm{D}\left(F_{1}\right)\right) \longrightarrow \mathrm{D}(\mathrm{D}(\operatorname{ker}(f))) \longrightarrow 0 .
$$

Therefore, we have the exact sequence

$$
\mathrm{D}\left(\mathrm{D}\left(F_{1}\right)\right) \longrightarrow \mathrm{D}\left(\mathrm{D}\left(F_{0}\right)\right) \longrightarrow \mathrm{D}(\mathrm{D}(M)) \longrightarrow 0 .
$$

We obtain the following diagram

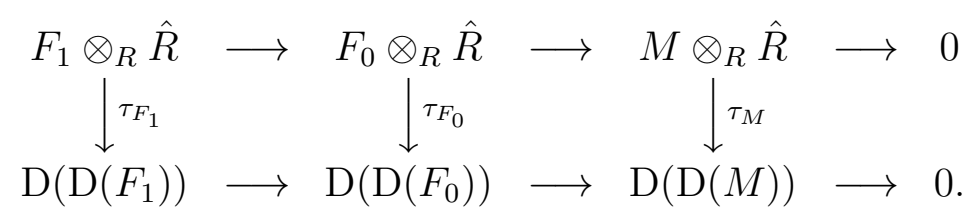

Now, by using [11, Lemma 3.59], we get that $\sigma_{F_{0}}$ and $\sigma_{F_{1}}$ are isomorphism and therefore $\tau_{F_{0}}$ and $\tau_{F_{1}}$ are also isomorphism. So, $\tau_{M}$ is an isomorphism.

Case 2. Either $\operatorname{ker}(f)=0$ or $\operatorname{ker}(g)=0$. In view of the above argument, it is obvious. 
The following example shows that Theorem 2.9 iii) does not hold for b.b.C.M. $R$-modules.

Example 2.10. Let $(R, \mathfrak{m})$ be a Gorenstein local ring of dimension zero and $F$ a nonfinitely generated free $R$-module. Then the canonical module of $R$ is $R=E_{R}(R / \mathfrak{m})$ and $F$ is a b.b.C.M. $R$-module. Suppose that $\tau_{F}$ is an isomorphism. This means that $F$ is Matlis reflexive and so by [4, Proposition 1.3], we have an exact sequence

$$
0 \longrightarrow L \longrightarrow F \longrightarrow A \longrightarrow 0,
$$

where $A$ is an Artinian $R$-module and $L$ a finitely generated $R$-module. Therefore, $\mu_{R}^{0}(\mathfrak{m}, F)$ is finite. This is a contradiction.

The following corollary is a generalization of Theorem 2.9.

Corollary 2.11. Let $(R, \mathfrak{m})$ be a Cohen-Macaulay local ring of dimension d and let $K$ be the canonical module of the completion of $R$. For all integers $t=0,1, \ldots, d$ and all $R$-modules $M$ of dimension $t$ which is such that $\mathfrak{m} M \neq M$ and there exists an $M$-sequence $x_{1}, \ldots, x_{t}$ we have

i) $\operatorname{Ext}_{R}^{d-t}(M, K)$ is an $R$-module of dimension $t$ and $x_{1}, \ldots, x_{t}$ is an $\operatorname{Ext}_{R}^{d-t}(M, K)$ sequence,

ii) $\operatorname{Ext}_{R}^{i}(M, K)=0$ for all $i \neq d-t$, and

iii)if $M$ is finitely generated, then there exists an isomorphism $M \otimes_{R} \hat{R} \longrightarrow$ $\operatorname{Ext}_{R}^{d-t}\left(\operatorname{Ext}_{R}^{d-t}(M, K), K\right)$ which in the case $d=t$ is just the natural isomorphism from $M \otimes_{R} \hat{R}$ into the bidual of $M$ with respect to $K$.

Proof. We obtain i) and ii) from Lemma 2.2 and Proposition 2.5.

iii) For $t=d$, the assertion follows from Theorem 2.9. By [9, page 140, Lemma 2(i)], we deduce that

$$
\operatorname{Ext}_{R}^{d-t}\left(\operatorname{Ext}_{R}^{d-t}(M, K), K\right) \cong \operatorname{Hom}_{R / \mathbf{x} \mathbf{R}}\left(\operatorname{Hom}_{R / \mathbf{x R}}(M, K / \mathbf{x} K), K / \mathbf{x} K\right)
$$

for an $R$-sequence $\mathbf{x}$ of length $d-t$ which is contained in $\operatorname{Ann}_{R}(M)$. Since $\operatorname{dim}_{R / \mathbf{x} R}(M)=t$ and $\operatorname{dim} R / \mathbf{x} R=t$, so $M$ is a maximal Cohen-Macaulay $R / \mathbf{x} R$ module. The module $K / \mathbf{x} K$ is the canonical module of $\hat{R} / \mathbf{x} \hat{R}$. Therefore, by Theorem 2.9 and above isomorphism, there is a natural isomorphism

$$
M \otimes_{R / \mathbf{x} R} \widehat{R / \mathbf{x} R} \longrightarrow \operatorname{Ext}_{R}^{d-t}\left(\operatorname{Ext}_{R}^{d-t}(M, K), K\right) .
$$

In view of $\widehat{R / \mathbf{x} R} \cong \hat{R} / \mathbf{x} \hat{R} \cong R / \mathbf{x} R \otimes_{R} \hat{R}$, we obtain the result. 


\section{Some Characterizations for Complete big Cohen-Macaulay MODULES OF FINITE INJECTIVE DIMENSION}

We need to recall some definitions.

Definition 3.1. Let $R$ be a Noetherian ring and let $M$ be an $R$-module.

i) The small support, or little support, of $M$ denoted by $\operatorname{supp}_{R}(M)$, is defined by

$$
\operatorname{supp}_{R}(M)=\left\{\mathfrak{p} \in \operatorname{Spec}(R) \mid \operatorname{depth}_{R_{\mathfrak{p}}}\left(M_{\mathfrak{p}}\right)<\infty\right\} .
$$

It is clear that $\operatorname{supp}_{R}(M) \subseteq \operatorname{Supp}_{R}(M)$; if $M$ is finitely generated, then these two sets are equal, but in general this need not be the case.

ii) A filtration of $\operatorname{Spec}(R)[\mathbf{1 6}, 1.1]$ is a descending sequence $\mathcal{F}=\left(F_{i}\right)_{i \geq 0}$ of subsets of $\operatorname{Spec}(R)$, so that

$$
F_{0} \supseteq F_{1} \supseteq \cdots \supseteq F_{i} \supseteq F_{i+1} \cdots,
$$

with the property that, for each $i \geq 0$, each member of $\partial F_{i}=F_{i} \backslash F_{i+1}$ is a minimal member of $F_{i}$ with respect to inclusion. We say that $\mathcal{F}$ admits $M$ if $\operatorname{Supp}_{R}(M) \subseteq F_{0}$. Suppose $\mathcal{F}$ is a filtration of $\operatorname{Spec}(R)$ that admits $M$. The Cousin complex $C(\mathcal{F}, M)$ for $M$ with respect to $\mathcal{F}$ has the form

$$
0 \stackrel{d^{-2}}{\longrightarrow} M \stackrel{d^{-1}}{\longrightarrow} M^{0} \stackrel{d^{0}}{\longrightarrow} M^{1} \longrightarrow \cdots \longrightarrow M^{n} \stackrel{d^{n}}{\longrightarrow} M^{n+1} \longrightarrow \cdots
$$

with $M^{n}=\oplus_{\mathfrak{q} \in \partial F_{n}}\left(\operatorname{coker} d^{n-2}\right)_{\mathfrak{q}}$ for all $n \geq 0$. The homomorphisms in this complex have the following properties: for $m \in M$ and $\mathfrak{q} \in \partial F_{0}$, the component of $d^{-1}(m)$ in $M_{\mathfrak{q}}$ is $m / 1$; for $n>0, x \in M^{n-1}$ and $\mathfrak{q} \in \partial F_{n}$, the component of $d^{n-1}(x)$ in $\left(\operatorname{coker} d^{n-2}\right)_{\mathfrak{q}}$ is $\bar{x} / 1$, where ${ }^{-}: M^{n-1} \longrightarrow$ coker $d^{n-2}$ is the canonical epimorphism. The fact that such a complex can be constructed is explained in $[\mathbf{1 6}, 1.3]$.

In the following proposition, we obtain some characterizations for complete big Cohen-Macaulay modules.

Proposition 3.2. Let $(R, \mathfrak{m})$ be a Noetherian local ring and $M$ a nonzero complete $R$-module. Denote by $\mathcal{D}(R)=\left(D_{i}\right)_{i \geq 0}$ (resp. $\left.\mathcal{H}(M)=\left(H_{i}\right)_{i \geq 0}\right)$ the dimension filtration of $\operatorname{Spec}(R)$ (resp. M-height filtration of $\operatorname{Spec}(R)$ ) i.e. $D_{i}=\{\mathfrak{p} \in \operatorname{Spec}(R) \mid$ $\operatorname{dim}(R / \mathfrak{p}) \leq \operatorname{dim}(R)-i\}$ (resp. $\left.H_{i}=\left\{\mathfrak{p} \in \operatorname{Supp}_{R}(M) \mid \mathrm{ht}_{M_{\mathfrak{p}}}\left(\mathfrak{p} R_{\mathfrak{p}}\right) \geq i\right\}\right)$. Then the following conditions are equivalent:

i) $M$ is a b.C.M. R-module with respect to an s.o.p. for $R$.

ii) $\operatorname{dp}_{R}(M)=\operatorname{dim}_{R}(M)=\operatorname{ht}_{M}(\mathfrak{m})=\operatorname{depth}_{R}(M)=\operatorname{dim}(R)$.

iii) $\operatorname{depth}_{R}(M)=\operatorname{dim}(R)$. 
iv) $M$ is a b.b.C.M. R-module.

v) $C(\mathcal{D}(R), M)$ is exact.

vi) Both the complexes $C(\mathcal{D}(R), M)$ and $C(\mathcal{H}(M), M)$ are isomorphic and $C(\mathcal{H}(M), M)$ is exact.

vii) $C(\mathcal{H}(M), M)$ is exact and $\mathrm{ht}_{M}(\mathfrak{m})=\operatorname{dim}(R)$.

Proof. $i) \Rightarrow i i)$ This is Definition and Remark $2.1 \mathrm{vi}$ ).

$i i) \Rightarrow i i i)$ It is clear.

$i i i) \Rightarrow i v)$ By $\left[\mathbf{1 8}\right.$, Proposition 10.1], for every $\mathfrak{p} \in \operatorname{supp}_{R}(M)$, we have

$$
\operatorname{depth}_{R}(M) \leq \operatorname{depth}_{R_{\mathfrak{p}}}\left(M_{\mathfrak{p}}\right)+\operatorname{dim}(R / \mathfrak{p}) \leq \operatorname{ht}(\mathfrak{p})+\operatorname{dim}(R / \mathfrak{p}) \leq \operatorname{dim}(R) .
$$

Therefore, $\operatorname{depth}_{R_{\mathfrak{p}}}\left(M_{\mathfrak{p}}\right)+\operatorname{dim}(R / \mathfrak{p})=\operatorname{dim}(R)$, for all $\mathfrak{p} \in \operatorname{supp}_{R}(M)$. On the other hand, since $M$ is complete, we have $\mathfrak{m} M \neq M$. Now, by [20, Theorem 3.3], we deduce that $M$ is b.b.C.M.

$i v) \Rightarrow v)$ This follows from [16, Corollary 3.7].

$v) \Rightarrow v i)$ This follows from $[\mathbf{1 7}$, Theorem 3.6].

$v i) \Rightarrow v i i) M$ is b.b.C.M. by [16, Corollary 3.7]. Hence, by Definition and Remark $2.1 \mathrm{vi})$, we have $\mathrm{ht}_{M}(\mathfrak{m})=\operatorname{dim}(R)$.

vii $\Rightarrow$ iii) Since $M$ is a complete $R$-module, $\mathfrak{m} M \neq M$. Therefore by Definition and Remark 2.1 ii), $\operatorname{depth}_{R}(M)$ is finite, So $\mathfrak{m} \in \operatorname{supp}_{R}(M)$. Hence, by [17, Corollary 3.5], we have $\operatorname{depth}_{R}(M)=\operatorname{ht}_{M}(\mathfrak{m})$.

$i v) \Rightarrow i$ ) It is clear.

Lemma 3.3. Let $(R, \mathfrak{m})$ be a Cohen-Macaulay local ring and $M$ a b.b.C.M. $R$ module of finite injective dimension. Then, for every $i>\operatorname{dim}_{R}(M)$, we have $\mu_{R}^{i}(\mathfrak{m}, M)=0$.

Proof. We prove the lemma by induction on $\operatorname{depth}(R)$. Let $\operatorname{depth}(R)=0$. Then $\mathfrak{m} \in \operatorname{Ass}(R)$. Thus we get an exact sequence

$$
0 \longrightarrow R / \mathfrak{m} \longrightarrow R \longrightarrow C \longrightarrow 0
$$

which implies the following exact sequence

$$
\operatorname{Ext}_{R}^{i}(R, M) \longrightarrow \operatorname{Ext}_{R}^{i}(R / \mathfrak{m}, M) \longrightarrow \operatorname{Ext}_{R}^{i+1}(C, M),
$$

for each $i \geq 0$. Let $r=\operatorname{injdim}_{R}(M)$. If $r>0$, then $\operatorname{Ext}_{R}^{r}(R, M)=0$, and consequently $\operatorname{Ext}_{R}^{r}(R / \mathfrak{m}, M)=0$. Since $\operatorname{dim}(R)=0, \mathfrak{m}$ is the unique prime ideal of $R$. Hence $\operatorname{inj}_{\operatorname{dim}}(M)<r$ by [2, Corollary 3.1.12], which is a contradiction. The 
assertion follows from $r=0$. Now suppose, inductively, that $\operatorname{depth}(R)>0$, and the result has been proved for the smaller value. Since $\operatorname{depth}(R)>0$, there exists $x \in \mathfrak{m}$ which is an $R$-sequence and $M$-sequence. Since $M$ is a b.b.C.M. $R$-module, $M / x M$ is a b.b.C.M. $R / x R$-module by [15, Lemma 2.3]. Therefore, in view of Definition and Remark 2.1 vi), we have

$$
\operatorname{dim}_{R / x R}(M / x M)=\operatorname{dim}(R / x R)=\operatorname{dim}(R)-1=\operatorname{dim}_{R}(M)-1 .
$$

Also, it follows from $[\mathbf{9}$, p. 140, Lemma 2(i)] that

$$
\operatorname{Ext}_{R / x R}^{i}(R / \mathfrak{m}, M / x M) \cong \operatorname{Ext}_{R}^{i+1}(R / \mathfrak{m}, M)
$$

for all $i \geq 0$ and $\operatorname{injdim}_{R / x R}(M / x M)<\infty$. Thus, by inductive hypothesis, for $i>\operatorname{dim}_{R}(M)-1=\operatorname{dim}_{R / x R}(M / x M)$, we have $\operatorname{Ext}_{R}^{i+1}(R / \mathfrak{m}, M)=0$. The assertion follows from this.

We are now ready to state and prove the main result of this section.

Theorem 3.4. Let $(R, \mathfrak{m})$ be a Noetherian local ring and $M$ a nonzero complete $R$-module. Let $\mathcal{D}(R)$ (resp. $\mathcal{H}(M)$ ) be the dimension filtration of $\operatorname{Spec}(R)$ (resp. $M$-height filtration of $\operatorname{Spec}(R))$. Then the following conditions are equivalent:

i) $C(\mathcal{D}(R), M)$ is a minimal injective resolution for $M$.

ii) $C(\mathcal{H}(M), M)$ is a minimal injective resolution for $M$ and $\operatorname{ht}_{M}(\mathfrak{m})=\operatorname{dim}(R)$.

iii) $\operatorname{ht}_{M}(\mathfrak{m})=\operatorname{dim}(R)$ and for every $\mathfrak{p} \in \operatorname{supp}_{R}(M), \mu_{R}^{i}(\mathfrak{p}, M) \neq 0$ if and only if $i \neq \mathrm{ht}_{M_{\mathfrak{p}}}\left(\mathfrak{p} R_{\mathfrak{p}}\right)$.

iv) $\mu_{R}^{i}(\mathfrak{m}, M)=0$ if and only if $i \neq \operatorname{dim}(R)$.

v) $M$ is a b.b.C.M. R-module of finite injective dimension.

vi) $M$ is a b.C.M. R-module with respect to a system of parameters for $R$ and $M$ has finite injective dimension.

vii) $\operatorname{depth}_{R}(M)=\operatorname{dim}(R)$ and $M$ has finite injective dimension.

viii) $\operatorname{injdim}_{R}(M)=\operatorname{depth}_{R}(M)=\operatorname{ht}_{M}(\mathfrak{m})=\operatorname{dim}_{R}(M)=\operatorname{dim}(R)=\operatorname{depth}(R)$.

Proof. $i) \Leftrightarrow i i$ ) This follows from Proposition 3.2.

ii $) \Rightarrow$ iii) Let $\mathfrak{p} \in \operatorname{supp}_{R}(M)$. We denote by $\mathcal{H}(M)_{\mathfrak{p}}$ the filtration $\left(F_{i}\right)_{i \in \mathbb{N}_{0}}$ of $\operatorname{Spec}\left(R_{\mathfrak{p}}\right)$, where $F_{i}=\left\{\mathfrak{q} R_{\mathfrak{p}} \mid \mathfrak{q} \in H_{i}\right.$ and $\left.\mathfrak{q} \subseteq \mathfrak{p}\right\}$. Then, by [12, Theorem 3.5], we have

$$
C\left(\mathcal{H}(M)_{\mathfrak{p}}, M_{\mathfrak{p}}\right) \cong C(\mathcal{H}(M), M)_{\mathfrak{p}}
$$


So, $\operatorname{injdim}_{R_{\mathfrak{p}}}\left(M_{\mathfrak{p}}\right) \leq \mathrm{ht}_{M_{\mathfrak{p}}}\left(\mathfrak{p} R_{\mathfrak{p}}\right)$. On the other hand, since $C(\mathcal{H}(M), M)$ is exact, it follows from $\left[\mathbf{1 7}\right.$, Corollary 3.5] that $\mathrm{ht}_{M_{\mathfrak{p}}}\left(\mathfrak{p} R_{\mathfrak{p}}\right)=\operatorname{depth}_{R_{\mathfrak{p}}}\left(M_{\mathfrak{p}}\right)$. Therefore $\operatorname{injdim}_{R_{\mathfrak{p}}}\left(M_{\mathfrak{p}}\right)=\operatorname{depth}_{R_{\mathfrak{p}}}\left(M_{\mathfrak{p}}\right)$. This ends this part.

iii) $\Rightarrow$ ii) Use the notation

$$
0 \stackrel{b^{-2}}{\longrightarrow} M \stackrel{b^{-1}}{\longrightarrow} B^{0} \longrightarrow B^{1} \longrightarrow \cdots \longrightarrow B^{n} \stackrel{b^{n}}{\longrightarrow} B^{n+1} \longrightarrow \cdots
$$

for the Cousin complex $C(\mathcal{H}(M), M)$. By, [17, Corollary 3.5], $C(\mathcal{H}(M), M)$ is exact. Let $n \in \mathbb{N}_{0}$ and (coker $\left.b^{n-2}\right)_{\mathfrak{q}}$ be the nonzero direct summand of $B^{n}$. It is enough to show that (coker $\left.b^{n-2}\right)_{\mathfrak{q}}$ is injective as an $R_{\mathfrak{q}}$-module. Note that every injective $R_{\mathfrak{q}}$-module is an injective $R$-module.

We have $\operatorname{ht}_{M}(\mathfrak{q})=n$ and $\mathfrak{q} \in \operatorname{Supp}_{R} M$. Hence, by [17, Corollary 2.6], $\left(\operatorname{coker} b^{n-2}\right)_{\mathfrak{q}}$ is $R_{\mathfrak{q}}$-isomorphic to $\mathrm{H}_{\mathfrak{q} R_{\mathfrak{q}}}^{n}\left(M_{\mathfrak{q}}\right)$, the n-th local cohomology module of $M_{\mathfrak{q}}$ with respect to the maximal ideal of the $R_{\mathfrak{q}}$. Since $\mathrm{H}_{\mathfrak{q} R_{\mathfrak{q}}}^{n}\left(M_{\mathfrak{q}}\right) \neq 0, \mathfrak{q} R_{\mathfrak{q}} M_{\mathfrak{q}} \neq M_{\mathfrak{q}}$, and so $\mathfrak{q} \in \operatorname{supp}_{R} M$. The minimal injective resolution

$$
0 \longrightarrow M_{\mathfrak{q}} \longrightarrow E^{0} \longrightarrow E^{1} \longrightarrow \cdots
$$

induces the following complex

$$
0 \longrightarrow \Gamma_{\mathfrak{q} R_{\mathfrak{q}}}\left(E^{0}\right) \longrightarrow \Gamma_{\mathfrak{q} R_{\mathfrak{q}}}\left(E^{1}\right) \longrightarrow \cdots \longrightarrow \Gamma_{\mathfrak{q} R_{\mathfrak{q}}}\left(E^{n}\right) \longrightarrow \Gamma_{\mathfrak{q} R_{\mathfrak{q}}}\left(E^{n+1}\right) \longrightarrow \cdots .
$$

By the assumption, we have $\mu_{R}^{i}\left(\mathfrak{q} R_{\mathfrak{q}}, M_{\mathfrak{q}}\right)=0$ for $i \neq n$. We obtain $\Gamma_{\mathfrak{q} R_{\mathfrak{q}}}\left(E^{i}\right)=0$ for every $i \neq n$. Therefore, we have

$$
\Gamma_{\mathfrak{q} R_{\mathfrak{q}}}\left(E^{n}\right) \cong \mathrm{H}_{\mathfrak{q} R_{\mathfrak{q}}}^{n}\left(M_{\mathfrak{q}}\right) .
$$

Hence, $\mathrm{H}_{\mathfrak{q} R_{\mathfrak{q}}}^{n}\left(M_{\mathfrak{q}}\right)$ is injective as an $R_{\mathfrak{q}}$-module.

$i i i) \Rightarrow i v$ ) The claim follows from the fact that $\mathfrak{m} \in \operatorname{supp}_{R}(M)$ (see the proof of Proposition 3.2 vii) $\Rightarrow$ iii)).

$i v) \Rightarrow v$ ) By assumption and Remark 2.3 iii), $\operatorname{depth}_{R}(M)=\operatorname{dim}(R)=\operatorname{injdim}_{R}(M)$. So, the claim follows from Proposition 3.2.

$v) \Rightarrow v i)$ This is clear.

$v i) \Rightarrow v i i)$ This follows from Definition and Remark 2.1 vi).

vii $) \Rightarrow$ viii) By Proposition 3.2, $\operatorname{depth}_{R}(M)=\mathrm{ht}_{M}(\mathfrak{m})=\operatorname{dim}_{R}(M)=\operatorname{dim}(R)$. Also, we have $\operatorname{depth}_{R}(M) \leq \operatorname{injdim}_{R}(M)$. So

$$
\operatorname{depth}_{R}(M)=\operatorname{ht}_{M}(\mathfrak{m})=\operatorname{dim}_{R}(M)=\operatorname{dim}(R) \leq \operatorname{injdim}_{R}(M) .
$$

The assertion follows from Remark 2.3 iv). 
viii $) \Rightarrow$ iii) Proposition 3.2 yields that $M$ is b.b.C.M.. Since $\operatorname{depth}(R)=\operatorname{dim}(R)$, $R$ is Cohen-Macaulay and therefore $R$ is catenary. Therefore by $[\mathbf{2 1}$, Proposition 2.6], $M_{\mathfrak{p}}$ is b.b.C.M. as an $R_{\mathfrak{p}}$-module. So, from Definition and Remark $2.1 \mathrm{vi}$ ), $\operatorname{depth}_{R_{\mathfrak{p}}}\left(M_{\mathfrak{p}}\right)=\mathrm{ht}_{M_{\mathfrak{p}}}\left(\mathfrak{p} R_{\mathfrak{p}}\right)=\operatorname{dim}_{R_{\mathfrak{p}}}\left(M_{\mathfrak{p}}\right)=\operatorname{dim}\left(R_{\mathfrak{p}}\right)$. On the other hand we know that $\operatorname{inj}_{\operatorname{dim}_{R_{\mathfrak{p}}}}\left(M_{\mathfrak{p}}\right)<\infty$. So by Lemma 3.3, $\mu_{R_{\mathfrak{p}}}^{i}\left(\mathfrak{p} R_{\mathfrak{p}}, M_{\mathfrak{p}}\right)=0$ for all $i>\operatorname{dim}_{R_{\mathfrak{p}}}\left(M_{\mathfrak{p}}\right)$. Therefore $\mu_{R}^{i}(\mathfrak{p}, M) \neq 0$ if and only if $i \neq \mathrm{ht}_{M_{\mathfrak{p}}}\left(\mathfrak{p} R_{\mathfrak{p}}\right)$. Note that $\mu_{R_{\mathfrak{p}}}^{i}\left(\mathfrak{p} R_{\mathfrak{p}}, M_{\mathfrak{p}}\right)=$ $\mu_{R}^{i}(\mathfrak{p}, M)$ for all $i$.

\section{Some Characterizations For Finite type COMPlete BiG}

\section{Cohen-MaCaulay modules of Finite injeCtive Dimension}

In this section, we present some characterizations for a Gorenstein $\hat{R}$-module $M$.

Lemma 4.1. Let $(R, \mathfrak{m})$ be a Noetherian local ring and $M$ an $R$-module. Suppose that $x \in \mathfrak{m}$ is both $R$-regular and $M$-regular. Then:

i) If $M$ is a b.b.C.M. R-module and $\operatorname{inj}_{\operatorname{dim}}(M)=\operatorname{depth}(R)$, then

$$
\operatorname{injdim}_{R / x R}(M / x M)=\operatorname{injdim}_{R}(M)-1 .
$$

ii) $\mu_{R}^{i}(\mathfrak{m}, M)=\mu_{R / x R}^{i-1}(\mathfrak{m} / x R, M / x M)$ for all $i \geq 1$.

Proof. i) By [19, Corollary 3.3.6(i)], we have injdim $\operatorname{ind}_{R R}(M / x M) \leq \operatorname{injdim}_{R}(M)-$ $1=\operatorname{depth}(R)-1$.

It is clear that $M / x M$ is a b.b.C.M. as an $R / x R$-module. So, by Definition and Remark 2.1 vi), we have

$$
\operatorname{depth}(R)-1 \leq \operatorname{dim}(R / x R)=\operatorname{depth}_{R / x R}(M / x M) \leq \operatorname{injdim}_{R / x R}(M / x M) .
$$

We can deduce the result from the above facts.

ii) This follows from $[\mathbf{9}$, p. 140, Lemma 2(i)].

Now we are able to prove our main result of this section.

Theorem 4.2. Let $(R, \mathfrak{m})$ be a Noetherian local ring of dimension $d$ and $M a$ nonzero $R$-module. The following conditions are equivalent:

i) $M$ is complete and big Cohen-Macaulay with respect to a system of parameters $x_{1}, \ldots, x_{d}$ such that $\operatorname{injdim}_{R} M$ and $\mu_{R}^{d}(\mathfrak{m}, M)$ are finite.

ii) $M$ is a Gorenstein $\hat{R}$-module.

iii) $M$ is complete and for all Cohen-Macaulay $R$-module $L$ of dimension $t$, we have 
a) $\operatorname{Ext}_{R}^{i}(L, M)=0$ for all $i \neq d-t$, and

b) $\operatorname{Ext}_{R}^{d-t}\left(\operatorname{Ext}_{R}^{d-t}(L, M), M\right) \cong L \otimes_{R} F$, where $F$ is a finitely generated free $\hat{R}$-module.

Proof. $i) \Rightarrow i i)$ Since $M$ is a complete $R$-module and $\hat{M}$ is an $\hat{R}$-module, $M$ has an $\hat{R}$-module structure. From Remark $2.3 \mathrm{iv)}$ and v), $\operatorname{injdim}_{R}(M)=\operatorname{depth}(R)$ and $R$ is a Cohen-Macaulay local ring. Also, Proposition 3.2 implies that $M$ is a b.b.C.M. $R$-module. Hence, by Lemma 4.1,

$$
\mu_{R}^{d}(\mathfrak{m}, M)=\mu_{R /\left(x_{1}, \ldots, x_{d}\right) R}^{0}\left(\mathfrak{m} /\left(x_{1}, \ldots, x_{d}\right), M /\left(x_{1}, \ldots, x_{d}\right) M\right)
$$

and

$$
\operatorname{injdim}_{R /\left(x_{1}, \ldots, x_{d}\right) R}\left(M /\left(x_{1}, \ldots, x_{d}\right) M\right)=\operatorname{injdim}_{R}(M)-d=0 .
$$

Therefore, by Matlis decomposition theorem, we get the following $R /\left(x_{1}, \ldots, x_{d}\right) R$ isomorphism.

$$
M /\left(x_{1}, \ldots, x_{d}\right) M \cong\left(E_{R /\left(x_{1}, \ldots, x_{d}\right) R}(R / \mathfrak{m})\right)^{\mu_{R}^{d}(\mathfrak{m}, M)}
$$

Since $E_{R /\left(x_{1}, \ldots, x_{d}\right) R}(R / \mathfrak{m})$ is finitely generated as an $R /\left(x_{1}, \ldots, x_{d}\right) R$-module, it turns out that $M /\left(x_{1}, \ldots, x_{d}\right) M$ is a finitely generated $R /\left(x_{1}, \ldots, x_{d}\right) R$-module. Now, from the natural isomorphism

$$
R /\left(x_{1}, \ldots, x_{d}\right) R \cong \hat{R} /\left(x_{1}, \ldots, x_{d}\right) \hat{R}
$$

we conclude that $M /\left(x_{1}, \ldots, x_{d}\right) M$ is a finitely generated $\hat{R} /\left(x_{1}, \ldots, x_{d}\right) \hat{R}$-module. Thus, there exist $m_{1}, \ldots, m_{t}$ in $M$ such that $M=\Sigma_{i=1}^{t} \hat{R} m_{i}+\mathfrak{m} M$. It is easy to see that $M$ is complete as an $\hat{R}$-module with respect to $\mathfrak{m} \hat{R}$-adic topology. Also, the finitely generated $\hat{R}$-module $\sum_{i=1}^{t} \hat{R} m_{i}$ is complete with respect to $\mathfrak{m} \hat{R}$-adic topology. So, by Remark $2.3 \mathrm{i}$ ), we have $M=\Sigma_{i=1}^{t} \hat{R} m_{i}$, that is, $M$ is a finitely generated $\hat{R}$-module.

Let $0 \longrightarrow M \longrightarrow E^{\bullet}$ be an injective resolution of $M$ as an $\hat{R}$-module. Since any injective $\hat{R}$-module is injective as an $R$-module, we have

$$
\begin{aligned}
\operatorname{Ext}_{R}^{i}(R / \mathfrak{m}, M) & \cong H^{i}\left(\operatorname{Hom}_{R}\left(R / \mathfrak{m}, E^{\bullet}\right)\right) \\
& \cong H^{i}\left(\operatorname{Hom}_{R}\left(R / \mathfrak{m}, \operatorname{Hom}_{\hat{R}}\left(\hat{R}, E^{\bullet}\right)\right)\right) \\
& \cong H^{i}\left(\operatorname{Hom}_{\hat{R}}\left(\hat{R} / \mathfrak{m} \hat{R}, E^{\bullet}\right)\right) \\
& \cong \operatorname{Ext}_{\hat{R}}^{i}(\hat{R} / \mathfrak{m} \hat{R}, M)
\end{aligned}
$$


Hence $\mu_{R}^{i}(\mathfrak{m}, M)=\mu_{\hat{R}}^{i}(\mathfrak{m} \hat{R}, M)$ for all $i \geq 0$. On the other hand, by Theorem 3.4, we have $\mu_{R}^{i}(\mathfrak{m}, M)=0$ for all $i \neq d$ and $\mu_{R}^{d}(\mathfrak{m}, M) \neq 0$. So the claim follows from [13, Theorem 3.11].

ii $\Rightarrow$ iii) It is easy to see that $M$ is a complete $R$-module with respect to m-adic topology. Since $M$ is a Gorenstein $\hat{R}$-module, $\hat{R}$ is a Cohen-Macaulay ring by [13, Theorem 3.11]. So $R$ is a Cohen-Macaulay ring.

Let $K$ be the canonical module of $\hat{R}$. As $\hat{R}$-modules, $M$ is isomorphic to $\bigoplus_{\mu_{\hat{R}}^{d}(\mathfrak{m} \hat{R}, M)} K$ by [14, Corollary 2.7]. So, the claim (a) follows from Corollary $\left.2.11 \mathrm{ii}\right)$.

We obtain

$$
\begin{aligned}
\operatorname{Ext}_{R}^{d-t}\left(\operatorname{Ext}_{R}^{d-t}(L, M), M\right) & \cong \operatorname{Ext}_{R}^{d-t}\left(\oplus_{\mu_{\hat{R}}^{d}(\mathfrak{m} \hat{R}, M)} \operatorname{Ext}_{R}^{d-t}(L, K), M\right) \\
& \cong \oplus_{\mu_{\hat{R}}^{d}(\mathfrak{m} \hat{R}, M)} \operatorname{Ext}_{R}^{d-t}\left(\operatorname{Ext}_{R}^{d-t}(L, K), M\right) \\
& \cong \oplus_{\left(\mu_{\hat{R}}^{d}(\mathfrak{m} \hat{R}, M)\right)} \operatorname{Ext}_{R}^{d-t}\left(\operatorname{Ext}_{R}^{d-t}(L, K), K\right) .
\end{aligned}
$$

From Corollary 2.11 iii), we have

$$
\begin{aligned}
\operatorname{Ext}_{R}^{d-t}\left(\operatorname{Ext}_{R}^{d-t}(L, M), M\right) & \cong \oplus_{\left(\mu_{\hat{R}}^{d}(\mathfrak{m} \hat{R}, M)\right)^{2}}\left(L \otimes_{R} \hat{R}\right) \\
& \cong L \otimes_{R}\left(\oplus_{\left(\mu_{\hat{R}}^{d}(\mathfrak{m} \hat{R}, M)\right)^{2}} \hat{R}\right) .
\end{aligned}
$$

This ends the proof of $\mathrm{b}$ ).

iii) $\Rightarrow i$ ) Let $L=R / \mathfrak{m}$. Since $\operatorname{dim}_{R} L=0, L$ is Cohen-Macaulay of dimension zero. Then a) and Remark 2.3 iii) implies that $\operatorname{depth}_{R}(M)=\operatorname{injdim}_{R}(M)=d$, and so by Proposition 3.2, $M$ is b.b.C.M. On the other hand b) yields that

$$
\operatorname{dim}_{R / \mathfrak{m}}\left(\operatorname{Ext}_{R}^{d}\left(\operatorname{Ext}_{R}^{d}(R / \mathfrak{m}, M), M\right)\right)
$$

is finite. We set $V=\operatorname{Ext}_{R}^{d}(R / \mathfrak{m}, M)$. Then $V=\oplus_{i \in I} F_{i}$ such that $F_{i}=R / \mathfrak{m}$ for all $i \in I$. So, $\operatorname{Ext}_{R}^{d}\left(\operatorname{Ext}_{R}^{d}(R / \mathfrak{m}, M), M\right)=\prod_{i \in I} V_{i}$ such that $V_{i}=V$ for all $i \in I$. Thus $I$ is finite, and so $\operatorname{dim}_{R / \mathfrak{m}} V$ is finite. The result follows as required.

\section{REFERENCES}

[1] M.P. Brodmann and R.Y. Sharp, Local cohomology: an algebraic introduction with geometric applications, Cambridge Studies in Advanced Mathematics, 60, Cambridge University Press, Cambridge, 1998.

[2] W. Bruns and J. Herzog, Cohen-Macaulay rings, Cambridge Studies in Advanced Mathematics, 39, Cambridge University Press, Cambridge, 1998.

[3] D. Eisenbud, Commutative algebra. With a view toward algebraic geometry, Graduate Texts in Mathematics, 150, Springer-Verlag, New York, 1995. 
[4] E. Enochs, Flat covers and flat cotorsion modules, Proc. Amer. Math. Soc., 92(2) (1984), 179-184.

[5] H.B. Foxby, Gorenstein modules and related modules, Math. Scand., 31 (1972), 267-284.

[6] H.B. Foxby, On the $\mu^{i}$ in a minimal injective resolution II, Math. Scand., 41(1) (1977), 19-44.

[7] A. Grothendieck, Local cohomology, Lect. Notes Math., 41, Springer-Verlag, 1967.

[8] J. Herzog and E. Kunz et al., Der kanonische Modul eines Cohen-Macaulay-Rings, Lect. Notes Math., 238, Springer-Verlag, 1971.

[9] H. Matsumura, Commutative ring theory, Cambridge Studies in Advanced Mathematics, 8, Cambridge University Press, Cambridge, 1986.

[10] I. Reiten, The converse to a theorem of Sharp on Gorenstein modules, Proc. Amer. Math. Soc., 32 (1972), 417-420.

[11] J. Rotman, Introduction to homological algebra, Academic Press, 1979.

[12] R.Y. Sharp, The Cousin complex for a module over a commutative Noetherian ring, Math. Z., 112 (1969), 340-356.

[13] R.Y. Sharp, Gorenstein modules, Math. Z., 115 (1970), 117-139.

[14] R.Y. Sharp, On Gorenstein modules over a complete Cohen-Macaulay local ring, Quart. J. Math. Oxford Ser., 22(2) (1971), 425-434.

[15] R.Y. Sharp, Cohen-Macaulay properties for balanced big Cohen-Macaulay modules, Math. Proc. Cambridge Philos. Soc., 90(2) (1981), 229-238.

[16] R.Y. Sharp, A Cousin complex characterization of balanced big Cohen-Macaulay modules, Quart. J. Math. Oxford (2), 33 (1982), 471-485.

[17] R.Y. Sharp and Z.M. Tang, On the structure of Cousin complexes, J. Math. Kyoto Univ., 33(1) (1993), 285-297.

[18] A.M. Simon, Some homological properties of complete modules, Math. Proc. Cambridge Philos. Soc., 108(2) (1990), 231-246.

[19] J. Strooker, Homological questions in local algebra, London Math. Soc. Lecture Note Ser., 145, Cambridge Univ. Press, (1990).

[20] S. Zarzuela, Systems of parameters for non-finitely generated modules and big Cohen-Macaulay modules, Mathematika, 35(2) (1988), 207-215.

[21] S. Zarzuela, Chain conditions arising from the study of non-finitely generated modules over commutative rings, Lecture Notes in Pure and Appl. Math., 153, Dekker, New York, (1994), 249-256.

M.A. Esmkhani, Department of Mathematics, Zanjan University, P.O. Box 45195313, ZANJAN, IrAN.

E-mail address: esmkhani@znu.ac.ir 
M. Tousi, Department of Mathematics, Shahid Beheshti University, Tehran, Iranand-Institute for Studies in Theoretical Physics and Mathematics, P.O. Box 193955746, TEHran, IRAN.

E-mail address: mtousi@ipm.ir 